Service social

\title{
Culture sportive et violence faite aux femmes
}

\section{Nathaly Gagnon}

Volume 44, numéro 2, 1995

Visages de la violence

URI : https://id.erudit.org/iderudit/706692ar

DOI : https://doi.org/10.7202/706692ar

Aller au sommaire du numéro

Éditeur(s)

École de service social de l'Université Laval

ISSN

1708-1734 (numérique)

Découvrir la revue

Citer cet article

Gagnon, N. (1995). Culture sportive et violence faite aux femmes. Service social, 44(2), 35-56. https://doi.org/10.7202/706692ar

\section{Résumé de l'article}

Cet article est un essai critique qui tente de dégager une perspective d'analyse sociale nouvelle pour une problématique qui, elle, ne l'est pas. Les questions suivantes sont soulevées: $\mathrm{Y}$ a-t-il des rapports possibles entre la socialisation masculine, la culture sportive et la violence à l'égard des femmes Existe-t-il une jonction entre "masculinité et sport" et "masculinité et violence" envers les femmes» La socialisation andro centrique qui domine la culture sportive actuelle contribue-t-elle à la violence envers les femmes Cette recherche, subventionnée par le CRSH, espère démontrer que la culture sportive contribue à la naturaliser " la violence et, ce faisant, perpétue la violence faite aux femmes. Le défi consiste donc à construire une vision de l'inacceptabilité devant la culture sportive en tant que lieu d'apprentissage et de la légitimation de la violence masculine. 


\section{Culture sportive et violence faite aux femmes}

NATHALY GAGNON

Politologue et professeure agrégée Département des sciences du loisir Université Concordia

On ne saurait s'intéresser à l'histoire, à la politique et aux relations humaines sans se rendre compte du rôle immense que la violence n'a cessé de jouer dans les affaires et dans le comportement des hommes. Il paraît assez surprenant, à première vue, que la violence, malgré les nombreuses analyses consacrées à ses causes et aux événements qui la déclenchent, puisse encore être considérée comme une chose «normale» et de ce fait négligée. C'est sans doute que les tendances perceptibles de la conjoncture actuelle préconisent encore deux visions populaires de la violence: celle qui s'exerce contre les femmes est vue comme un «état de fait inéluctable», "naturel », alors que celle qui constitue le symbole de la puissance masculine au sein de plusieurs sports est jugée «légitime», "applaudie» et «anticipée». Cette double vision présente aussi l'éradication de ces deux formes de violence comme un exploit extraordinaire qui correspondrait, en quelque sorte, à une véritable transformation de la «nature» 
humaine. Or, le défi proposé ici est de construire une vision de l'inacceptabilité devant la culture sportive en tant que lieu d'apprentissage et de la légitimation de la violence masculine.

Le présent article constitue un essai critique à partir de la recension des écrits afin de dégager une perspective d'analyse sociale nouvelle pour une problématique qui, elle, ne l'est pas. Cette recherche, subventionnée par le Conseil de recherche en sciences humaines (1994-1996), vise essentiellement les objectifs suivants: identifier les rapports possibles entre la socialisation masculine, la culture sportive et la violence qui s'exerce à l'égard des femmes, particulièrement dans le contexte domestique / familial ; susciter une analyse théorique, si de tels rapports existent, sur la jonction «masculinité et sports » / "masculinité et violence envers les femmes». Enfin, parmi les questions de recherche sous-jacentes, notons en particulier celle qui est pertinente pour une réflexion liée à l'intervention sociale: la socialisation androcentrique qui domine la culture sportive actuelle contribue-t-elle à la violence à l'égard des femmes?

Le présent texte, au risque de frustrer certaines lectrices et certains lecteurs, ne propose que des conclusions partielles. Ultérieurement, I'analyse de contenu des entretiens en cours permettra de générer une production discursive pour comprendre quelle place occupe le sport et comment, d'une part, il contribue à l'identification sociosexuelle de l'homme et, d'autre part, il «permet » à celui-ci, le cas échéant, de violenter sa conjointe.

L'étude est née d'une constatation assez élémentaire, pour ne pas dire banale: la violence envers les femmes survient surtout durant le temps "libre». Ce phénomène, qui n'a jamais fait l'objet d'une étude scientifique, est pourtant signalé dans les statistiques canadiennes concernant la violence envers les femmes. Par exemple, une étude récente indique que $83 \%$ des incidents de violence faite aux femmes survenaient à la résidence de la victime, pendant que $9 \%$ survenaient à la résidence d'une personne connue de la victime. On estime également qu'environ $75 \%$ des actes de violence survenaient durant la soirée ou la nuit et près de $45 \%$ durant la fin de semaine (Johnson, 1990: 174). Ce temps «libre» est généralement consacré aux loisirs, et le loisir qui de loin rallie la majorité de la population canadienne est la télévision (Postman, 1985; Young, 1990). Or, les heures d'écoute les plus populaires sont surtout liées aux sports professionnels : hockey (La Soirée du Hockey est l'émission la plus regardée au Canada), football, baseball (Cantelon et Gruneau, 1988: 181), particulièrement lorsqu'on étudie les habitudes d'écoute des hommes (Gloor, 1992 ; Young, 1990). Ces émissions sont présentées 
en soirée durant la semaine, et elles occupent un espace dominant des plages horaires des réseaux de télévision privés durant la fin de semaine.

Finalement, il est à signaler que la présente recherche, de type exploratoire, est la première du genre au Canada et au Québec. La recherche préliminaire effectuée en 1991 révélait en effet que ce sujet n'avait pas encore fait l'objet d'étude scientifique, et les références bibliographiques relatives aux concepts de violence conjugale, socialisation, culture sportive, sports de masse ne faisaient ressortir aucun lien entre des incidents de violence envers les femmes et des événements sportifs liés à la culture sportive dominante. Pourtant, à la même époque, au sein des milieux d'intervention interrogés par I'auteure et qui agissent respectivement auprès des femmes violentées et des conjoints agresseurs, on soupçonnait que de tels liens existent sans toutefois pouvoir les expliquer, les confirmer, les nier et surtout sans jamais les avoir analysés.

\section{CONTEXTE SOCIAL}

La nature endémique et profondément enracinée de la violence faite aux femmes dans notre société est liée à notre système de valeurs et à nos attitudes, elles-mêmes tributaires de plusieurs siècles d'oppression systématique. Ce phénomène ne peut être compris qu'en rapport avec le contexte social de la vie des femmes d'hier et d'aujourd'hui et des valeurs traditionnellement associées aux rôles masculins et féminins. Parmi les caractéristiques fondamentales de cette socialisation, il faut souligner l'éducation androcentrique, le renforcement des valeurs et des rôles traditionnels liés à l'appartenance sexuelle, ainsi que la violence associée à la culture sportive. Force est de constater que l'évaluation de la portée sociale et politique du sport dans notre société est très récente, notamment en ce qui concerne notre compréhension de la manière dont les sports contribuent à reproduire les inégalités sociales et à renforcer la domination du genre masculin. Cette domination se caractérise, notamment, par son monopole de l'agressivité et de la violence en général. En effet, comme le souligne Welzer-Lang (1991), les groupes sociaux chargés d'exercer la violence sont des groupes dominés par les hommes (police, armée, justice). La violence légitime, celle que l'on considère comme «normale», est donc une violence à symbolique masculine. Les coups, inconsciemment, viennent dans la maison rappeler cette chaîne de pouvoirs. L'homme violent n'est plus un homme isolé, mais 
devient alors, en quelque sorte, représentant dans son contexte privé du pouvoir général. Nous ajouterions qu'il existe une autre forme de violence légitime, soit celle qui est omniprésente dans les sports professionnels les plus populaires: hockey, football.

\section{Hypothèses de recherche}

La recherche pose des questions qui s'articulent autour de la fonction sociale du genre, du phénomène endémique de la violence envers les femmes, du phénomène de la violence entre hommes, en particulier celle qui est fort bien tolérée, voire applaudie, soit la violence sportive. Pour ce faire, nous nous inspirons des perspectives socioanthropologiques féministes et posons les trois hypothèses suivantes:

- À un premier niveau, la culture sportive serait l'un des lieux centraux de la production sociale de la masculinité. Le sport est une institution androcentrique, non seulement dans ses formes, mais dans les valeurs et les normes comportementales qu'il promeut et, ultimement, "naturalise » tant au sein du jeu lui-même qu'au sein des hiérarchies sociales.

- À un second niveau, tributaire du premier, les comportements ainsi engendrés recevraient une approbation sociale, contribuant ainsi à maintenir des modèles de privilèges masculins $v s$ des modèles de subordination des femmes, structures qui existent en dehors du sport lui-même.

- Enfin, le sport contribuerait en fait à la formation de modèles historiques du pouvoir masculin sur les femmes, d'où l'importance d'expliquer le rôle du sport dans la reproduction / transformation des relations contemporaines entre les hommes et les femmes.

Il faut convenir avec Deslauriers qu'on ne peut plus étudier les transformations sociales et les microsystèmes sociaux avec un instrument qui mise uniquement sur la régularité, la stabilité et le grand nombre. "Il faut s'approcher du terrain, se faire plus inductif et se laisser imprégner de l'air du temps»(Deslauriers, 1991: 5). À cet égard, I'abondance de publicité visant à contrer la violence faite aux femmes lors des Super Bowl télévisés de 1993 et 1994 devrait nous faire réfléchir: sans se préoccuper des répercussions sociales d'un tel geste ni attendre que des chercheurs s'intéressent sérieusement à la question, les promoteurs ont décidé d'agir comme si une corrélation existait véritablement entre cet événement sportif majeur et la recrudescence de la violence envers les femmes. Il faut surtout retenir de ce geste qu'il s'inscrit dans la foulée des efforts des femmes pour s'opposer de toutes les manières possibles à la violence qui s'exerce contre elles. 


\section{ÉLÉMENTS DE PROBLÉMATIQUE}

\section{Violence faite aux femmes et sport: une intuition}

Ce sont deux articles parus dans le quotidien The Gazette de Montréal en mars 1991 qui ont suscité la présente réflexion (Capuzzo, 1991 ; Schwarts, 1991). On y décrivait en substance ce que I'expérience de l'auteure au sein de groupes de femmes avait permis de supposer: un lien probable entre des incidents de violence contre les femmes, particulièrement en contexte familial, et la présence de I'agresseur / conjoint violent à un match sportif, à titre de spectateur ou de téléspectateur. Décrivant la réalité qui prévaut aux États-Unis, on notait que le football et le basket-ball sont directement liés à la violence contre les femmes. On signalait notamment que le dimanche du Super Bowl constitue la journée la plus violente de l'année pour les femmes. On rapportait également que $25 \%$ des cas traités dans un centre pour hommes violents impliquaient une violence envers leurs conjointes reliée aux sports. On affirmait enfin que I'assistance aux sports professionnels est l'un des déclencheurs principaux de ce type de violence domestique. Mais ce qui avait le plus attiré notre attention à l'époque est le fait que les articles révélaient l'absence de toute étude sérieuse sur un lien possible entre les sports et la violence domestique, surtout au Canada, et ce, même si les intervenants et intervenantes dans le milieu ne s'étonnaient pas de la possibilité d'un tel rapport.

\section{Violence envers les femmes: un phénomène bien documenté}

La définition dont s'inspire cet essai est tirée d'un texte produit par le Conseil consultatif canadien sur la situation de la femme intitulé $L a$ violence faite aux femmes par les hommes: la brutalité de l'inégalité:

la violence faite aux femmes est un problème complexe qui comprend la violence physique, sexuelle, psychologique et économique contre les femmes et qui est tributaire des structures, des valeurs et des mesures sociales, économiques et politiques qui musellent les femmes dans notre société, favorisent la discrimination fondée sur le sexe et maintiennent I'inégalité des femmes (Canada, 1991:5).

Cette définition a l'avantage de lier les abus dont les femmes sont victimes à l'inégalité de leur situation dans notre société ainsi qu'aux croyances, attitudes et valeurs sociales qui font en sorte qu'on 
ferme les yeux sur la violence faite aux femmes. Cette façon de concevoir la violence à l'égard des femmes mène inévitablement à la conclusion qu'il faut, pour trouver des solutions efficaces, modifier les valeurs traditionnelles qui régissent les rapports entre les sexes. Les médias canadiens rapportent quotidiennement des cas d'agression physique perpétrée contre des femmes, notamment par des personnes qui avaient la confiance des victimes. La fréquence des incidents de cette nature rapportés par les médias devrait, à elle seule, inciter la plupart des Canadiens à s'interroger sur l'ampleur effective du phénomène de la violence faite aux femmes et sur les causes qui la déclenchent. De nombreux rapports gouvernementaux ont pourtant étudié la violence masculine: MacLeod (1980 et 1987), Badgley (1984), Fraser (1984) et plus récemment le document controversé - par l'ampleur du phénomène qu'il soulignait - produit par le Comité canadien sur la violence faite aux femmes (Canada, 1993).

En fait, tous et toutes s'entendent pour affirmer que la gravité de la situation dépasse de loin le nombre de cas signalés. Les recherches montrent effectivement que la plupart des femmes victimes de violence ne portent pas plainte à la police et que moins de $50 \%$ des femmes violentées physiquement en avisent la police (Canada, 1991; 1993 ; Sansfaçon, 1991, 1992). Par conséquent, le taux réel d'agressions et les circonstances qui les ont provoquées demeurent inconnus et ne figurent pas dans les statistiques criminelles officielles ni dans les reportages médiatiques. Ces mêmes études montrent également que la répugnance des femmes à signaler ces attaques violentes est liée à la crainte de représailles susceptibles de mettre leur vie en danger ou de les exposer à d'autres gestes violents, au manque de confiance dans les forces policières et les tribunaux, sans compter le sentiment de culpabilité et de gêne lié aux incidents de violence (Dobash et Dobash, 1992).

Le système de déclaration uniforme de la criminalité qui tient des statistiques sur les incidents de nature criminelle qui se produisent au Canada ne fait état que des crimes qui sont signalés à la police et tous les cas de femmes violentées figurent sous la rubrique plus vaste des agressions en général. Le système ne contient pas de renseignement descriptifs sur les rapports entre les victimes et les agresseurs ni sur les circonstances entourant l'infraction qui permettraient de repérer les cas de violence en contexte familial. Une recherche menée par Welzer-Lang note également la survie de stéréotypes tenaces, comme l'association commune entre le fait qu'un homme consomme de l'alcool pour oublier ou pour se laisser aller à exprimer ses sentiments et au fait qu'il s'autorise à frapper sa femme. 
Ce genre d'association provient de l'adhésion consciente ou inconsciente aux stéréotypes masculins:

La croyance que la virilité est associée à I'alcool et à la force et que la force est associée à la violence [...]. L'association violence $=$ alcool est aussi pratique pour les hommes violents. Elle permet à certains de se présenter comme irresponsables ou bien elle permet à leurs compagnes d'accepter les excuses de leurs maris ou compagnons (Welzer-Lang, 1991: 53).

Par ailleurs, les études spéciales réalisées auprès des maisons $d^{\prime}$ accueil pour femmes violentées, des centres d'aide aux victimes $d^{\prime}$ agression sexuelle et $d^{\prime}$ autres organismes de première ligne ne fournissent qu'une idée du nombre de victimes qui sollicitent I'aide de ces services sans véritablement préciser les motifs invoqués entourant I'agression (Canada, 1991, 1993; Sansfaçon, 1992). En fait, I'agresseur est souvent socialement cautionné pour ses «pertes de contrôle ». On remarque des sentences dérisoires ou même des annulations de procès pour "délais déraisonnables" (ministère de la Sécurité publique, 1988), sans parler du peu d'ordonnances pour les services d'aide à l'agresseur: tous ces faits sanctionnent en soi la violence et $n^{\prime}$ incitent pas les agresseurs à modifier leurs comportements violents ni à déceler les situations qui les provoquent. Or, certaines des causes engendrant cette violence demeurent obscures et la présente recherche se veut une contribution à l'élargissement de nos connaissances sur des phénomènes et des événements sociaux potentiellement déclencheurs de violence.

La nécessité d'explorer théoriquement et empiriquement les divers déclencheurs de la violence a été soulignée par Murdock et McCron (1978), Dobash et Dobash (1979), Théberge (1989), Welzer-Lang (1992) et d'autres qui affirment que de meilleures recherches sont nécessaires pour mieux comprendre la prévalence de la violence envers les femmes. Par ailleurs, selon Dobash et Dobash (1992), les sources principales de conflit conduisant aux attaques violentes de la part de conjoints agresseurs sont la possessivité, la jalousie, les attentes des hommes concernant le rôle des femmes, leur croyance au droit de punir «leur» conjointe en cas de non-conformité à ce qu'ils attendent d'elle et l'importance pour les hommes de maintenir ou d'exercer leur position d'autorité. Nombreuses sont les femmes qui ressentent un sentiment de honte et la crainte de représailles, ce qui les confine au silence pendant des années. La répugnance des femmes à rapporter la violence dont elles sont victimes est souvent exacerbée par les institutions légales, médicales et sociales dont les actions révèlent un legs puissant de politiques et de pratiques qui 
explicitement ou implicitement acceptent ou ignorent la violence masculine ou, pire encore, blâment la victime et la désignent comme responsable pour trouver des solutions et pour éliminer cette violence (Dobash et Dobash, 1992; Edwards, 1989). Même si ces dernières années certaines thérapies radicales et féministes ont tenté de défier I'approche classique, elles ont souvent été perverties par les pratiques traditionnelles. De la même manière, bien que les pratiques récentes en travail social aient tenté de surpasser les approches individualisées en mettant l'accent par exemple sur l'écosystème, ce sont surtout les relations interpersonnelles qui dominent l'intervention et non pas les contextes plus larges (politiques, culturels et sociaux) qui sont justement au cœur des problèmes sociaux (Hall, 1992 ; Worell et Remer, 1992).

Les écrits consultés ont également montré que la violence exercée contre les femmes risque plus de survenir durant leurs rapports intimes avec des hommes. Qu'il s'agisse d'assaut sexuel, de viol ou $\mathrm{d}^{\prime}$ assaut physique, les femmes ont plus à craindre de leurs pères, époux et amis que d'étrangers, et elles ne courent presque aucun risque d'être attaquées par d'autres femmes (Dobash et Dobash, 1992 ; Gelles et Straus, 1988). La violence sévère et systématique contre les femmes est associée presque exclusivement à une relation permanente avec un homme. En outre, la violence faite aux femmes au sein de l'espace domestique est un phénomène unique dans le monde de la violence. Ce n'est qu'en prison (ou dans une institution similaire) qu'un individu risque de subir de tels abus, une telle violence et ce genre de terreur persistante (Gagnon, 1989). Pour expliquer cette violence, il faut donc analyser les liens complexes tirés des multiples évidences empiriques de source historique et contemporaine. II émerge de cette analyse que cette violence est surtout le fait d'hommes et qu'elle survient dans le contexte de la domination sociale masculine (Yllö et Bograd, 1988). Il convient aussi de procéder à une étude concrète des actes, motifs, intentions et interprétations tant de ceux qui commettent la violence que de celles qui la subissent. Une analyse minutieuse de la violence et des contextes immédiats qui lui sont associés met en relief les croyances, la socialisation et les pratiques culturelles sexuées. Finalement, ces éléments sont alors confrontés avec les contextes plus larges des actions et des orientations institutionnelles qui, aujourd'hui comme hier, ont soutenu la répression violente exercée par les hommes sur les femmes et ont nié ou minimisé son impact sur ces dernières. 


\section{Socialisation et culture sportive : une analyse en émergence}

On encourage encore les garçons et les hommes à être agressifs, forts et dominateurs. À cet égard, la culture sportive constitue un lieu de socialisation dominant (Burton-Nelson, 1994 ; Whitson, 1990). Inversement, on s'attend à ce que les femmes soient plus passives, obéissantes et soumises, et leur exclusion de la culture sportive dominante ne peut pas être considérée comme une coïncidence. L'éducation sexiste ou androcentrique, le renforcement des valeurs et des rôles traditionnels ainsi que la violence associée à la culture sportive sont des caractéristiques fondamentales de la socialisation occidentale et constituent des phénomènes de mieux en mieux connus (Messner et Sabo, 1990). La contribution du sport masculin à la reproduction de I'hégémonie masculine dans la société est devenue pour plusieurs évidente. Cette affirmation comporte deux aspects centraux, essentiels à la présente recherche. Premièrement, le sport est considéré comme une institution mâle, non seulement en termes numériques, ce que plusieurs chercheurs ont déjà indiqué (MacKinnon, 1987 ; Messner, 1990 ; Messner et Sabo, 1990; Théberge, 1981), mais, de façon plus importante, par rapport aux valeurs et aux normes comportementales qu'il promeut et ultimement «naturalise», tant dans sa pratique que dans ses modes d'organisation hiérarchique (Dunning, 1986 ; Farrell, 1974 ; Hoch, 1972 ; Naison, 1972 ; Whitson, 1990). Deuxièmement, et à cause de ce qui précède, on peut avancer que I'attention sociale et surtout le statut social accordés aux comportements masculins présents dans le sport contribuent à confirmer les modèles de privilège masculin et de subordination féminine (en fait, les structures de domination) qui existent en dehors du sport lui-même (Atkinson, 1978; Kidd, 1990; Lenskyj, 1986; Sabo et Panepinto, 1990).

Il est donc permis d'affirmer que le sport est devenu l'un des sites centraux de la production sociale de la masculinité dans les sociétés caractérisées, d'une part, par un niveau d'éducation plus élevé et, d'autre part, par un déclin des activités sociales nécessitant une très grande force physique. De plus, le fait de posséder les attributs psychologiques et physiques associés à la réussite dans des compétitions athlétiques est maintenant devenu une exigence liée au statut social au sein des groupes d'adolescents et de pré-adolescents. Ironiquement, des auteurs comme Hoch (1972) ont identifié le sport comme "l'école du sexisme», tandis que Naison (1972) le considère comme une source institutionnelle de "l'idéologie de la domination mâle ». Dans son analyse de la signification culturelle du Super Bowl, 
Farrell (1974) n'hésite pas à faire des liens entre le sport, la masculinité, le sexisme et le militarisme.

Par ailleurs, Théberge (1989) note que la base sociale de la violence présente dans le sport et ses conséquences n'ont pas été véritablement étudiées, du moins sous I'angle de leur rapport avec la violence envers les femmes. Semyonov et Farbstein (1989) soutiennent que la relation causale joueur/spectateur est asymétrique: les joueurs affectent la violence chez les spectateurs, mais pas inversement. Weis (1986) suggère que l'agressivité des athlètes professionnels et la violence chez les spectateurs sont soulignées par les médias comme des événements "normaux». Les recherches de Philipps (1983 à 1987) et de Forry (1985) soulignent un lien entre le visionnement d'une agression à la télévision et l'augmentation du comportement agressif. Dès son utilisation dans le domaine du sport, la télévision s'est avérée un puissant véhicule de l'idéologie dominante et, selon Cantelon et Gruneau (1988), la recherche portant sur les effets qu'exerce le spectacle sur le public a constitué le filon dominant pour la recherche sur le sport et les médias. Ces auteurs révèlent eux aussi que I'émission La Soirée du Hockey est la plus regardée au Canada. On peut donc légitimement se demander quel est l'impact du message sur le spectateur, surtout quand on sait que le sport de masse rejoint un vaste auditoire aux caractéristiques démographiques clairement identifiables. Cependant, peu de chercheurs s'attardent à expliquer pourquoi un sport plutôt qu'un autre apparaît à l'écran, ou encore à cerner la place qu'occupe la violence dans la société. Le télé/spectateur et la télé/spectatrice finissent par "comprendre» que certaines manifestations, certains sports et un sexe en particulier ont plus d'importance que d'autres.

Un autre élément $d^{\prime}$ 'analyse à considérer est cette singulière similarité de l'écart entre l'application des lois et du Code criminel lorsqu'il s'agit de la violence dans les sports (Smith, 1983) et de la violence contre les femmes: les deux domaines sont pratiquement «immunisés » contre des charges criminelles significatives. Goldstein (1982) affirme que les sports enseignent et stimulent la violence comme le reflet des valeurs et attitudes de la culture dont ils sont partie intégrante. Messner (1988 à 1990), Talbot (1988), Bryson (1983 à 1988), Foley (1990), Sabo (1985) démontrent que les sports contribuent à la domination du genre masculin, à son monopole de l'agressivité et de la violence et certains analysent, par exemple, comment le football reproduit les inégalités sociales (Sabo et Panepinto, 1990; Burton-Nelson, 1994). Dans la foulée du procès médiatisé et de I'acquittement fort controversé de l'ancienne vedette du football américain O.J. Simpson, leur analyse mérite qu'on s'y attarde. 


\section{MASCULINITÉ/SPORT : MASCULINITÉ/VIOLENCE Le cas du football}

\section{Cadre analytique}

- Le rituel lié au football s'apparente aux rites initiatiques mâles primitifs.

- Le rituel initiatique découle d'une structure sociale et contribue à la construction de l'identité individuelle à partir d'une allocation des rôles.

- Le rituel initiatique reproduit à la fois la structure et I'éthique culturelle d'une société donnée.

- On qualifie de patriarcal un rituel contenant des éléments de socialisation sexuelle qui exprime et promeut des modèles de domination masculine.

- Les rituels primitifs mâles et le football possèdent plusieurs éléments en commun :

\section{RITUELS PRIMITIFS}

\section{Relation homme-garçon}

La relation initiant-initié : apprentissage des comportements, croyances, valeurs "appropriées » entre hommes uniquement.

\section{Conformité et contrôle}

Pression, menace, punition et récompense selon le niveau de conformité obtenu.

\section{Isolement social}

Les «initiés" sont tenus à l'écart des femmes et des filles. Alors que I'on prône les vertus masculines on dénigre ou ignore les femmes et la féminité.

\section{Respect de l'autorité mâle}

La relation initiant-initié est fortement hiérarchisée.

\section{Douleur}

Les rituels initiatiques sont accompagnés de douleur: celle infligée aux initiés pour tester leur endurance et leur courage et pour les différencier des autres groupes (hommes et femmes non initiés).

\section{RITUEL DU FOOTBALL}

\section{Relation homme-garçon}

La relation entraîneur-joueur est basée sur le même principe.

\section{Conformité et contrôle}

Les entraîneurs exercent des moyens similaires de contrôle sur les joueurs et exigent la conformité.

\section{Isolement social}

Le vestiaire ou le terrain de football sont réservés aux hommes ;

ces derniers s'entraînent, voyagent, $\mathrm{s}^{\prime}$ amusent entre eux. Lorsque les femmes sont présentes, elles occupent une position subalterne : cheerleader, hôtesse, admiratrice...

\section{Respect de l'autorité mâle}

Différence de statut au sein de l'équipe des entraîneurs, et entre les entraîneurs et les joueurs, les équipes professionnelles et les autres, les vedettes et les autres joueurs...

\section{Douleur}

Le football est fondé sur l'assaut physique; la douleur et les blessures sont le lot quotidien des joueurs. 


\section{MASCULINITÉ/SPORT: MASCULINITÉ/VIOLENCE}

\section{Les liens entre la virilité et le corps}

Certains analystes avancent qu'apprendre, grâce à la pratique de sports, à utiliser son corps de manière vigoureuse et sans se gêner pour occuper tout l'espace nécessaire, de même qu'apprendre à associer de tels comportements au fait d'être un homme, a contribué, pour beaucoup de garçons, à déterminer leurs attentes dans les rapports sociaux qu'ils entretiennent avec des femmes et avec $\mathrm{d}^{\prime}$ autres hommes. D'autres chercheurs notent que le sport, en tant que bastion masculin, a servi de lieu important dans la construction de la solidarité mâle, une institution qui encourage des hommes à s'identifier à d'autres hommes et fournit des occasions régulières de renouveler de telles identifications. C'est l'ensemble de ces dynamiques qui suggère le rôle prépondérant du sport dans la reproduction de l'hégémonie masculine.

\section{"Transformer un garçon en homme »}

Il s'agit bien là d'une croyance ancienne: la capacité du sport à transformer un garçon en un "vrai » homme. C'est en quelque sorte une association historique entre les muscles et la morale (Mangan, 1981; Weber, 1970)! En effet, on a longtemps considéré que les pratiques athlétiques permettaient d'acquérir une présence physique, un courage stoïque dans l'endurance de douleur et une capacité de prendre des décisions sous pression; tout cela était décrit simplement comme faisant partie intégrante de l'accomplissement de la virilité (Whitson, 1990). Par ailleurs, les pratiques athlétiques ont toujours été l'occasion de maximiser et de célébrer, entre hommes, les différences entre les hommes et les femmes et les différences entre certaines catégories d'hommes. La masculinité devient donc l'antithèse de ce qui n'est pas "féminin »ou, plus carrément, "efféminé » (Craib, 1987).

Par ailleurs, les normes mâles d'endurance physique et mentale sont elles-mêmes des constructions historiques. Connell (1983) affirme que cette présence au corps est cruciale pour le développement de l'identité mâle et suggère qu'apprendre à être un mâle c'est apprendre à projeter une présence physique imbue de pouvoir potentiel. En ce sens, le sport est donc un outil d'empowerment pour les jeunes hommes, précisément parce qu'il leur enseigne comment se servir de leur corps pour démontrer leur pouvoir par des combi- 
naisons de force et d'habileté. Si Connell a raison de prétendre que la connaissance de soi est fermement enracinée dans notre expérience d'incarnation, force est de reconnaître que cette dernière est partie intégrante de la reproduction des relations de genre, les garçons étant encouragés à éprouver leur corps, et donc eux-mêmes, de manière vigoureuse, dominante et sans se gêner pour occuper tout I'espace dont ils veulent disposer. Cela veut peut-être dire que les pratiques associées au corps sont au cœur de la construction sociale de la masculinité et de la féminité, et c'est précisément pourquoi le sport doit être inclus dans l'étude de la structure des relations de genre.

Par conséquent, nous suggérons que l'affirmation de soi et la confiance en soi, en tant que moyens de communication avec l'autre, s'incarnent par le développement de la force et de l'habileté physiques et par la capacité de dominer des adversaires dans des situations concurrentielles. Inversement, Young (1979) avance que I'exclusion des filles de cette culture sportive a historiquement nié aux femmes ce genre d'expériences formatrices, de sorte que leur sens incarné de soi tend à faire d'elles des êtres plutôt maladroits, assez fragiles et qui manquent de confiance. Bien que l'entrée des femmes dans plusieurs sports (quoique très réduite au sein de sports professionnels de confrontation comme le football) ait eu tendance à modifier cette description des femmes, elle demeure prépondérante chez les femmes adultes. Par ailleurs, même s'il existe aujourd'hui des femmes qui réalisent des exploits de force et $d^{\prime}$ habileté supérieurs à ceux accomplis par beaucoup d'hommes, la persistance de différences «normales» dans le processus d'empowerment demeure l'une des manières principales par lesquelles la supériorité des hommes est «naturalisée ». Les occasions quasi quotidiennes de célébrer la supériorité mâle à travers le Monday Night Football et La Soirée du Hockey peuvent être surtout importantes pour des hommes qui se sentent menacés par les changements contemporains dans des relations entre les sexes. Il faut se rappeler que les conjoints agresseurs d'aujourd'hui sont des adultes qui ont été socialisés à partir de ce schéma traditionnel. La condamnation sociale de la violence envers les femmes ne date que de quelques années et $\mathrm{n}^{\prime}$ a certainement pas connu d'équivalent en ce qui concerne une réflexion sociale de la violence légitime visible quotidiennement dans le sport professionnel.

Dans la culture occidentale contemporaine, la plupart des sports professionnels d'équipe «ritualisent»I'agression et lui permettent d'être reliée aux notions de compétitivité et de masculinité. Nous pouvons donc avancer que le sport est aussi devenu l'un des sites les plus importants des pratiques de «masculinisation » au sein de 
cultures où toutes les autres formes de prouesse physique ont perdu de leur valeur et où toute autre forme $d^{\prime}$ agression directe est officiellement illégale. L'importance que nous continuons d'attribuer aux sports d'équipe de type agressif dans notre hiérarchie des sports et I'admiration que nous accordons aux hommes qui excellent dans ces sports signifient que ces derniers constituent toujours des lieux importants de pratiques de masculinisation. Autrement dit, les sports majeurs continuent d'être des institutions par lesquelles la reproduction de la masculinité comme figure hégémonique et I'hégémonie masculine elle-même sont activement recréées. Ces sports sont typiquement des institutions où la force physique et la capacité de se battre sont célébrées, où la solidarité mâle, et surtout la solidarité parmi les mâles agressifs et dominants, est aussi célébrée, ce qui renforce les contraintes sur des garçons qui voudraient expérimenter $\mathrm{d}^{\prime}$ autres manières de devenir des hommes. Il est donc impératif de "déconstruire» ce lien entre empowerment et domination. Cela nécessitera que nous offrions aux garçons et aux filles des expériences similaires d'empowerment qui permettent de développer habileté et force physique par des sports non agressifs et que nous devrions célébrer ces qualités plus que les capacités d'agresser autrui.

Pour beaucoup de féministes, le sport a été identifié, assez justement, comme une activité suprêmement mâle et il a été plutôt esquivé, sauf quelques exceptions, tant dans la pratique que comme sujet d'investigation. Cependant, une telle attitude ne peut plus être soutenue, puisque si les femmes doivent comprendre les procédés de leur domination, c'est à leur risque et péril qu'elles ignorent le sport (MacKinnon, 1987). Ce dernier constitue une institution puissante par laquelle I'hégémonie masculine est construite et reconstruite, et c'est seulement en comprenant et en confrontant ces procédés que les femmes et les hommes peuvent espérer briser cette domination. En effet, le sport est devenu un outil de l'hégémonie masculine de deux façons: d'abord, en liant la masculinité aux habiletés valorisées dans notre société et, ensuite, en liant la masculinité à l'emploi sanctionné de l'agression, de la force et de la violence. Le fait de réserver le sport aux hommes a aussi pour effet d'inférioriser la féminité et les activités féminines.

On peut donc avancer que le sport sert à soutenir rituellement un aura de supériorité et de compétence masculine pour des habiletés publiquement acclamées, comme il soutient le monopole des hommes sur l'agression et la violence. L'inverse de ce processus mène à l'infériorisation des femmes et de leurs habiletés ainsi qu'à leur exclusion de la base ultime du pouvoir social, la force physique. 
La caractéristique principale des sports socialement valorisés est précisément qu'ils sont hautement visibles, c'est-à-dire qu'ils reçoivent une couverture médiatique d'envergure et qu'ils sont communément pratiqués dans presque toutes les localités, si bien que les enfants s'identifient à eux en tentant souvent d'imiter les équipes professionnelles et leurs idoles.

Une autre caractéristique importante est qu'au moins un certain nombre des activités sportives masculines auxquelles les gens sont continuellement exposés comportent de l'agression physique: ainsi les garçons apprennent que ces comportements sont acceptables, qu'ils peuvent les pratiquer et, de concert avec d'autres, qu'ils peuvent même les applaudir (Burton-Nelson, 1994). C'est surtout cet aspect rituel du sport qui renforce I'hégémonie masculine même si le sport offre un soutien social à d'autres valeurs telles que le nationalisme, l'identité ethnique et religieuse, la compétitivité, I'individualisme, etc. En fait, les événements sportifs majeurs célébrés de façon routinière et presque quotidienne sont ceux auxquels ne participent que des hommes (si l'on exclut la représentation marginale des femmes à titre, par exemple, de cheerleaders) et auxquels assistent des auditoires composés en majorité d'hommes.

Enfin, on peut sans doute affirmer que dans la société actuelle, certains sports - hockey, rugby, soccer, boxe, football - sont des enclaves de l'expression socialement acceptable, ritualisée et plus ou moins contrôlée de la violence physique et que cela confère un prestige social aux hommes qui peuvent se battre physiquement. Corrélativement, ces hommes ont tendance à développer un amour de la lutte physique et de considérer celle-ci comme une source centrale de signification et de satisfaction dans leur vie (Dunning, 1986).

\section{DÉMARCHES À VENIR ASSOCIÉES À LA PRÉSENTE RECHERCHE}

La première phase de cette recherche exploratoire consistait à définir les liens conceptuels potentiels entre la socialisation masculine à travers la culture sportive et la violence envers les femmes. Le présent texte fait état des réflexions à ce jour. La seconde phase de la recherche est basée sur des entretiens non directifs en profondeur obtenus auprès de soixante personnes divisées en quatre groupes distincts. D'abord des hommes réputés non violents et des conjoints agresseurs. Dans le cas du second groupe, les perceptions des incidents de violence auxquels ces hommes ont participé et leur interprétation des 
causes pourront mettre en évidence les différentes logiques sociales qui les génèrent. La dynamique de l'identification sociosexuelle, dont leurs réactions à la violence, et l'importance du sport dans leur vie seront analysées. Enfin, des entretiens semi-structurés avec des intervenantes de maisons d'hébergement pour femmes violentées et des intervenants auprès de conjoints agresseurs sont en cours et l'analyse finale de l'ensemble de ces entretiens, confrontée à I'analyse critique des divers éléments évoqués dans le présent document, sera publiée en juin 1996.

\section{CONCLUSION PARTIELLE}

Pouvoir, puissance, force, autorité, violence: ce ne sont là que des mots indicateurs des moyens que I'homme utilise afin de dominer; on les tient pour synonymes du fait qu'ils ont la même fonction. Mais I'histoire nous enseigne qu'il n'existe pas de gouvernement ou de mariage qui soient exclusivement fondés sur l'emploi des moyens de la violence. La forme de domination la plus despotique que I'humanité ait pu concevoir, celle des maîtres sur des esclaves qui leur furent toujours très supérieurs en nombre, ne reposait pas ellemême sur des moyens de contrainte particulièrement puissants, mais sur la supériorité de l'organisation, c'est-à-dire sur la solidarité organisée des maîtres. Les hommes isolés, qui ne peuvent avoir recours à I'appui de leurs semblables, n'ont jamais disposé d'un pouvoir suffisant pour se servir avec succès de la violence (Arendt, 1972: 160). Il en est de même de la violence envers les femmes. Les hommes ont créé des institutions millénaires qui leur ont permis de maintenir leur hégémonie sur les femmes de façon organisée, concertée et légitime: Église, mariage, politique, justice. Cette violence n'est donc pas plus bestiale qu'irrationnelle elle est, par nature, instrumentale. Comme tous les instruments, elle doit toujours être dirigée et justifiée par les fins qu'elle entend servir. Le véritable défi est donc de déterminer à quoi peut encore servir cette barbarie à l'aube du $21^{\mathrm{e}}$ siècle.

Toutes les personnes engagées en intervention sociale s'entendent sur la difficulté énorme de lutter contre des attitudes enracinées et institutionnalisées. À cet égard, les stratégies et tactiques des féministes visant un changement des comportements violents ont été, d'une part, ingénieuses et efficaces et, d'autre part, exposées à I'inexpérience $d^{\prime}$ un mouvement relativement nouveau, en lutte contre des adversaires possédant un passé long et «infâme". Les femmes ont contribué à l'opposition sociale à la violence en démontrant 
habileté, perspicacité et enthousiasme; elles ont été alimentées par une passion et une colère légitime contre la violence qu'elles subiss(ai)ent. Le défi le plus significatif dans le processus de changement social est précisément l'acte même de créer de nouvelles visions. Dès qu'une nouvelle idée se matérialise, il est difficile de s'imaginer comment il a pu en être autrement. Mais au moment de "lancer» cette nouvelle idée, il est difficile de s'imaginer comment elle se concrétisera. Il est donc impérieux de reconnaître que, si I'on convient généralement qu'il est inacceptable pour un homme d'exercer une violence sexuelle ou physique contre sa partenaire, cette croyance est très récente et dénote en fait un changement extraordinaire, d'envergure considérable eu égard à la façon de penser jusqu'à un passé récent.

Le processus de changement social et politique implique non seulement l'expression d'idées claires et nouvelles sur le monde en devenir, mais aussi la traduction de cette vision en action concrète de manière à véritablement créer le changement envisagé. Inévitablement, de telles actions entraînent des négociations et des luttes avec ceux qui possèdent une vision du monde différente, habituellement ancrée dans le monde qu'ils connaissent déjà et qui leur procure des avantages certains. Acquérir une nouvelle vision et agir pour la réaliser constituent le cœur des mouvements sociaux. Former une vision qui représente véritablement une solution de remplacement au statu quo constitue le cœur du changement social. Et lutter pour des changements qui transforment la vie de toutes les femmes constitue le cœur du projet féministe de société (Dobash et Dobash, 1992). Ces trois éléments sont réunis dans le mouvement des femmes qui s'opposent à la violence et qui défendent celles qui en sont les victimes.

Des échos de cette lutte se font entendre tous les jours dans les parlements, les médias, les conversations publiques et privées. Ce discours est novateur en ce sens qu'il construit une vision de «l'inacceptabilité » plutôt que de la tolérance de la violence mâle. En affirmant que les femmes ont le droit d'occuper l'espace public à titre de citoyennes, ce discours s'éloigne des notions précédentes selon lesquelles elles n'étaient que des épouses ou des mères dont l'existence était justifiée par leur capacité à répondre aux besoins d'autrui. Ce discours oppose également l'image d'une bonne épouse loyale à son époux en restant silencieuse sur les abus de ce dernier à l'image d'une citoyenne pourvue de droits (dont le droit à l'intégrité physique et morale) et de responsabilités. Cette vision et les actions qui l'accompagnent posent un défi tant aux hommes qui seraient violents 
qu'à ceux qui permettraient à leurs congénères de continuer à l'être en toute impunité.

Si I'on convient que le sport est un fief masculin farouchement gardé, n'est-il pas futile de ne s'attaquer qu'aux symptômes du problème social que constitue la violence envers les femmes? Les centres de femmes, les maisons d'hébergement, les groupes d'intervention auprès des conjoints agresseurs sont certes nécessaires mais, $d^{\prime}$ 'une part, ils sont insuffisants pour endiguer le problème et, d'autre part, ils n'ont pas d'impact sur le processus de socialisation qui contribue justement à perpétuer cette violence. Si I'on admet que la culture sportive est I'un des derniers bastions masculins, si I'on démontre que ce bastion est en fait l'un des lieux centraux de I'apprentissage des modèles hégémoniques de violence, n'est-il pas temps de repenser / confronter / analyser minutieusement non seulement la place qu'il occupe mais la manière dont il se reproduit?

\section{Références bibliographiques}

ARENDT, H. (1972). Du mensonge à la violence. Paris: Calmann-Lévy.

ARMS, R. et al. (1979). "Effects on the Hostility of Spectators of Viewing Aggressive Sports », Social Psychology Quarterly, vol. 42, n $3: 275-$ 279 .

ATKINSON, P. (1979). "Fitness, feminism and schooling», dans S. Delamont et L. Duffin (dir.), The 19th Century Woman: Her physical and Cultural World, p. 92-133. Londres: Chroom Helm.

ATHERTON, J. et R. SiBLey (dir.) (1988). "Le sport en Grande-Bretagne et aux États-Unis », Actes du Colloque CERCA, Nancy.

BANDurA, A. (1973). Aggression: A Social Learning Analysis. New Jersey: Prentice Hall.

BARRETt, M. (1987). "The concept of difference», Feminist Review, vol. 26 : 29-41.

BEAMISH, R. (1982). "Sport and the Logic of Capitalism», Sport, Culture and the Modern State. Toronto: Univ. of Toronto Press.

BenhabiB, S. (1992). Situating the Self. New York: Routledge.

BENJAMIN, J. (1988). The bonds of love: psychoanalysis, feminism, and the problem of domination. New York: Pantheon Books.

BergmaN, B. et al. (1988). "Aetiological and Precipitating Factors in Wife Battering », Acta Psychiatria Scandinavia, vol. 77, n 3 : 338-345.

BIRKE, L. et G. VINES (1987). "A sporting chance: the anatomy of destiny", Women's Studies International Forum, vol. 10, $\mathrm{n}^{\circ} 4: 337-347$.

BIRRELL, S. (1988). "Discourses on the gender / sport relationship », Exercise and Sport Science Reviews, vol.16:459-502. 
Bourdieu, P. (1978). "Pratiques sportives et pratiques sociales», Actes du VII Congrès international, HISPA, tome 1. Paris : INSEP.

BRUSH, L.D. (1990). "Violent Acts and Injurious Outcomes in Married Couples», Gender and Society, vol. 4, n $1: 56-67$.

BRYSON, L. (1983). "Sport and the Oppression of Women», Australian and New Zealand Journal of Sociology, vol. 19, $\mathrm{n}^{\circ} 3:$ 413-426.

BRYSON, L. (1987). "Sport and the Maintenance of Masculine Hegemony», Women's Studies International Forum, vol. 10, $\mathrm{n}^{\circ} 4: 349-360$.

BuRstow, B. (1992). Radical Feminist Therapy: Working in the Context of Violence. Londres: Sage.

Burton-Nelson, M. (1994). The Stronger Women Get, The More Men Love Football: Sexism and the American Culture of Sports. New York: Harcourt Brace \& Company.

CANADA (1991). La guerre contre les femmes. Rapport du Sous-comité permanent de la Chambre des Communes sur la condition féminine. Ottawa: Approvisionnements et Services, 2 volumes.

CANADA (1993). La violence faite aux femmes. Rapport du Comité canadien sur la violence faite aux femmes. Ottawa: Approvisionnements et Services.

CANTELON et GRUNEAU (1988). "La production des sports à la télévision ", Sport et pouvoir: les enjeux sociaux au Canada. Ottawa: Presses de I'Université d'Ottawa.

CAPuzzO, M. (1991). "Jokes about Wife-Beating abound in Jock Talk of Coaches, Players, Fans », The Gazette, Montréal, 8 mars.

COAKLeY, J. (1986). Sport in Society: Issues and Controversies. St. Louis: Times / Mirror Mosby.

COLBURN, K., Jr. (1986). «Deviance and Legitimacy in Ice-Hockey: A Theory of Violence», Sociological Quarterly, vol. 27, n $1: 63-74$.

COMMISSION DE POLICE DU QUÉBEC (1983). Analyse opérationnelle de la criminalité. Québec.

CONNELL, R.W. (1983). Which way is up? Essays on class, sex and culture. Sidney, Australia: Allen and Unwin.

CRAIB, I. (1987). "Masculinity and male dominance», Sociological Review, vol. 35, no $4: 721-743$.

DESLAURIERS, J.-P. (1991). Recherche qualitative : guide pratique. Montréal : McGraw-Hill.

DOBASH, R.E. et R.P. DOBASH (1979). Violence Against Wives: A Case Against the Patriarchy. New York: Free Press Macmillan.

DOBASH, R.E. et R.P. DOBASH (1992). Women, Violence and Social Change. Londres: Routledge.

DUNNING, E. (1986). "Sport as a Male Preserve: Notes on the Social Sources of Masculine Identity », Theory, Culture and Society, vol. 3, $\mathrm{n}^{\circ} 1$ : 79-90.

DUNNING, E. (1990). "Sociological Reflections on Sport, Violence and Civilization», Sociology of Sport, vol. 25, n $1:$ 65-82.

DUQUIN, M. (1984). "Power and authority: Moral consensus and conformity in sport », International Review for Sociology of Sport, vol. 19: 295-304. 
EdWards, S. (1989). Policing "Domestic 》 Violence. Londres: Sage.

ELIAS E. et N. DUNNING (1986). Quest for excitement: Sport and leisure in the civilizing process. New York: Blackwell.

FARRELL, W. (1974). The liberated man: Freeing men and their relationships with women. New York: Random House.

FOLEY, D. (1990). «The Great American Football Ritual: Reproducing Race, Class, Gender Inequality », Sociology of Sport Journal, vol. 7, $\mathrm{n}^{\circ} 2$ : 111-135.

FORRY, F.E. (1985). An Investigation of the Effects of Video Violence upon Male. Thèse de doctorat, Temple University.

GAGNON, N. (1989). Un vol organisé : La discrimination des femmes. Hull: Éditions Asticou, Collection Critiques.

Gelles, R.J. et M.A. Straus (1988). Intimate Violence: The Causes and Consequences of Abuse in American Families. New York: Simon and Schuster.

GlOOR, D. (1992). "Women Versus Men? The Hidden Differences in Leisure Activities », Loisir et Société / Society and Leisure, vol. 15, n $1: 39-60$.

GoldsteIN, J.H. (1982). "Sports Violence», National Forum, vol. 62, no 1 : 9-11.

GreENE, L. (1987). "Just Fun and Games: A Symbolic Analysis of Sports in Modern Societies», Human Mosaic, vol. 21, n 1/2: 44-56.

Greendorfer, S.L. (1981). "Race and Gender Differences in Children's Socialization into Sport», Research Quarterly, vol. 52:301-310.

HALL, M. (1992). Women and Empowerment. Washington: Hemisphere.

HANMER, J. et S. SAUNDERS (1984). Well-founded Fear: A Community Study of Violence to Women. Londres: Women's Research and Resource Center.

HARGREAVES, J. (dir.). (1982). Sport, Culture and Ideology. Londres : Routledge et Kegan Paul.

Hargreaves, J. (dir.). (1987). "The Body, Sport and Power Relations", Sociological Review Monograph, vol. 33 : 139-159.

HARVEY, J. et H. CANTELON (1988). Sport et pouvoir: les enjeux sociaux au Canada. Ottawa: Presses de I'Université d'Ottawa.

$\mathrm{HOCH}$, P. (1972). Rip off the big game. New York: Anchor Press.

JOHNSON, H. (1990). "Wife Abuse», Canadian Social Trends. Thompson Educational Publishing.

KIDD, B. (1990). "The Men's Cultural Centre: Sports and the Dynamic of Women's Oppression / Men's Repression », dans Messner et Sabo, p. 31-44.

KILLORAN, M. (1984). "Notes Towards a Metatheory of Wife Abuse», Journal of International and Comparative Social Welfare, vol. 1, $\mathrm{n}^{\circ} 1: 30-39$.

LAROUCHE, G. (1981). Agir contre la violence. Montréal: Les éditions de la Pleine Lune.

LENSKYJ, H. (1986). Out of Bounds : Women, Sport and Sexuality. Toronto: Women's Press.

LESTER, D. (1988). "Suicide and Homicide during Major Sport Events 1972$1984 »$, Sociology of Sport Journal, vol. 5, n $3: 285$. 
MACKINNON, C.A. (1987). "Women, Self-Possession and Sport», Feminism Unmodified, Harvard University Press.

MAGUIRE, J. (1986). "The Emergence of Football Spectating as a Social Problem 1880-1985», Sociology of Sport Journal, vol. 3, n 3 : 217-244.

MANGAN, J.A. (1981). Athleticism in the Victorian and Edwardian Public Schools. Cambridge, England: Cambridge University Press.

MESSNER, M.A. (1988). "Sports and Male Domination», Sociology of Sport Journal, vol. 5, no $3: 197-211$.

MESSNER, M.A. (1990). "When Bodies are Weapons: Masculinity and Violence in Sport », Sociology of Sport Journal, vol. 25, $\mathrm{n}^{\circ} 3:$ 203-220.

Messner, M.A. et D.F. SABO (dir.) (1990). Sport, Men and the Gender Order. A Critical Feminist Perspectives. Champaign, Illinois: Human Kinetics.

MINISTÈRE DE LA SÉCURITÉ PUBLIQUE (1988-1993). Violence conjugale : statistiques. Québec.

Murdock et MCCRON (1978). "Television and Teen Age Violence», New Society, vol. 46, $\mathrm{n}^{\circ} 845:$ 632-633.

NAISON, M. (1972). "Sports and the American Empire», Radical America, p. 95-110.

PHILIPPS, D.P. (1983). "The Impact of Mass Media Violence on U.S. Homicides », American Sociological Review, vol. 48, nº 4 : 560-568.

PHILIPPS, D.P. (1987). "Mass Media and Imitative Aggression», Social Science, vol. 72, $\mathrm{n}^{\circ}$ 2/4: 216-218.

Postman, N. (1985). Amusing Ourselves to Death. Penguin Books.

PRICE, L.S. (1989). Patterns of Violence in the Lives of Girls and Women: A Reading Guide. Vancouver: Women's Research Centre.

RAPOPORT, D. (1986). Television Sports Violence: Factors which Influence the Replay of Violence. San Francisco State University.

SABO, D. (1985). "Sport, Patriarchy, and Male Identity: New Questions about Men and Sport», Arena Review, vol. 9, n 2: 1-30.

$\mathrm{SABO}$, D. et J. Panepinto (1990). "Football rituals and the social reproduction of mascunality », dans M.A. Messner et D. Sabo, p. 115-126.

SANSFAÇON, D. (1991). La violence conjugale en morceaux. Ottawa: Solliciteur général.

SANSFAÇON, D. (1992). Rapport final de la recherche action sur l'intervention socio-judiciaire en matière de violence conjugale à Laval. Ottawa : Solliciteur général.

SANTONI, R. (1994). "On the Existential Meaning of Violence», Violence et coexistence humaine actes du II congrès mondial de l'ASEVICO, p. 53-68.

SCHWARTS, S. (1991). "Moods Aroused by Sports Carry Beyond the Arena », The Gazette, Montréal, 8 mars.

SEMYONOV et FARBSTEIN (1989). "Ecology of Sport Violence», Sociology of Sport Journal, vol. 6, $\mathrm{n}^{\circ} 1: 50-59$.

SMITH, M.D. (1983). Violence and Sport. Toronto: Butterworths.

SuGDEN, J.P. (1981). "The Sociological Perspective: The Political Economy of Violence in American Sport », ARENA, vol. 5, $\mathrm{n}^{\circ} 1: 57-62$. 
TALBOT, M. (1988). "Understanding the Relationships between Women and Sport », Sociology of Sport, vol. 23, $\mathrm{n}^{\circ} 1: 31-41$.

ThÉBERGE, N. (1981). "A Critique of Critique: Radical Feminist Writings on Sport », Social Forum, vol. 60:341-353.

ThéberGe, N. (1989). "Social Control and Women in Sport», dans J. Freeman (dir.), Women: A Feminist Perspective. Mountainview, CA : Mayfield.

WAlker, G. (1990). "Conceptual Politics of Struggle: Wife Battering, the Women's Movement and the State », Studies in Political Economy, vol. 33, automne: 63-90.

WeBER, E. (1970). "Pierre de Coubertin and the Introduction of Organized Sport into France », Journal of Contemporary History, vol. 5, $\mathrm{n}^{\circ} 2$ : $3-26$.

WeIS, K. (1986). "How the Print Media Affect Sports and Violence», Sociology of Sport, vol. 21, $\mathrm{n}^{\circ}$ 2/3 : 239-252.

WelZER-LANG, D. (1991). Les hommes violents. Paris: Lierre et Coudrier.

WeLZER-LANG, D. (1992). Arrête! Tu me fais mal. Montréal: VLB éditeur.

WhITSON, D. (1990). "Sport in the Social Construction of Masculinity », dans Messner et Sabo, p. 19-30.

WORELL, J. et P. Remer (1992). Feminist Perspectives in Therapy. New York: John Wiley et Sons.

YllÖ, K. et M. BOGRAD (dir.) (1988). Feminist Perspective on Wive Abuse. Newbury Park: Sage.

YOUNG, A. (1990). "Television Viewing», Canadian Social Trends. Thompson Educational Publishing. 